\title{
Diabetes Incidence and Long-Term Exposure to Air Pollution
}

ZORANA J. ANDERSEN, PHD ${ }^{1}$

Ole RaAsChOU-Nielsen, PHD ${ }^{1}$

Matthias Ketzel, PHD ${ }^{2}$

STEEN S. JENSEN, PHD 2

Martin Hvidberg, Bs ${ }^{2}$

OBJECTIVE-Animal and cross-sectional epidemiological studies suggest a link between air pollution and diabetes, whereas the limited prospective data show mixed results. We studied the association between long-term exposure to traffic-related air pollution and incidence of diabetes.

RESEARCH DESICN AND METHODS - We followed 57,053 participants of the Danish Diet, Cancer, and Health cohort in the Danish National Diabetes Register between baseline (1993-1997) and 27 June 2006. We estimated the mean levels of nitrogen dioxide $\left(\mathrm{NO}_{2}\right)$ at the residential addresses of the cohort participants since 1971 and modeled the association between $\mathrm{NO}_{2}$ and diabetes incidence with a Cox regression model, separately for two definitions of diabetes: all cases and a more strict definition where unconfirmed cases were excluded.

RESULTS-Over a mean follow-up of 9.7 years of 51,818 eligible subjects, there were 4,040 (7.8\%) incident diabetes cases in total and 2,877 (5.5\%) with confirmed diagnoses. Air pollution was not associated with all diabetes cases (hazard ratio 1.00 [95\% CI 0.97-1.04] per interquartile range of $4.9 \mu \mathrm{g} / \mathrm{m}^{3}$ mean $\mathrm{NO}_{2}$ levels since 1971), but a borderline statistically significant association was detected with confirmed cases of diabetes (1.04 [1.00-1.08]). Among confirmed diabetes cases, effects were significantly enhanced in nonsmokers (1.12 [1.05-1.20]) and physically active people (1.10 [1.03-1.16]).

CONCLUSIONS-Long-term exposure to traffic-related air pollution may contribute to the development of diabetes, especially in individuals with a healthy lifestyle, nonsmokers, and physically active individuals.

Diabetes Care 35:92-98, 2012

$\mathbf{T}$ he prevalence and incidence of type 2 diabetes are rising rapidly in both the developed and developing world, presenting one of the greatest contributors to the global burden of the disease (1). The diabetes epidemic is in large part attributable to established causes related to modern lifestyle including obesity, physical inactivity, and the growing aging populations (1). Environmental exposures linked to industrialization and urbanization, such as air pollution, have not been considered risk factors for diabetes until recently (2). In the U.S., the prevalence of

$\bullet \bullet \bullet \bullet \bullet \bullet \bullet \bullet \bullet \bullet \bullet \bullet \bullet \bullet \bullet \bullet \bullet \bullet \bullet \bullet \bullet \bullet \bullet \bullet \bullet \bullet \bullet \bullet \bullet \bullet \bullet \bullet \bullet \bullet \bullet \bullet \bullet \bullet \bullet \bullet \bullet \bullet \bullet \bullet \bullet \bullet \bullet \bullet \bullet$

From the ${ }^{1}$ Institute of Cancer Epidemiology, Danish Cancer Society, Copenhagen, Denmark; the ${ }^{2}$ Department of Environmental Science, Aarhus University, Roskilde, Denmark; the ${ }^{3}$ Section of Environmental Health, Department of Public Health, University of Copenhagen, Copenhagen, Denmark; the ${ }^{4}$ Department of Epidemiology, School of Public Health, Aarhus University, Aarhus, Denmark; and the ${ }^{5}$ Department of Cardiology, Centre for Cardiovascular Research, Aalborg Hospital, Aarhus University Hospital, Aalborg, Denmark.

Corresponding author: Zorana J. Andersen, zorana@cancer.dk.

Received 17 June 2011 and accepted 7 October 2011.

DOI: $10.2337 / \mathrm{dcll}-1155$

This article contains Supplementary Data online at http://care.diabetesjournals.org/lookup/suppl/doi:10 2337/dc11-1155/-/DCl.

K.O. and M.S. contributed equally to this work.

(C) 2012 by the American Diabetes Association. Readers may use this article as long as the work is properly cited, the use is educational and not for profit, and the work is not altered. See http://creativecommons.org/ licenses/by-nc-nd/3.0/ for details

diabetes correlated with the release of toxicants into the air (3), whereas diabetic people appeared more vulnerable than nondiabetic people to cardiovascular health effects associated with exposure to air pollution (4). Diabetes and cardiovascular diseases share many risk factors, and diabetic people are at a highly increased risk for heart or circulatory disorders (5). The central biological mechanisms of air pollution damage to the heart and blood vessels include inflammation (6), which is also believed to be involved in the promotion of insulin resistance and type 2 diabetes (7). An enhanced association between air pollution and inflammation, endothelial dysfunction, prothrombotic changes, and altered heart rate variability was found in diabetic people (6). A plausible biological mechanism of air pollution promoting diabetes was provided by Sun et al. (8), showing that exposure to particulate air pollution caused increased blood glucose, inflammation in adipose tissue, and insulin resistance in high-fat diet-fed mice. Recent study confirmed that prolonged exposure to air pollution leads to insulin resistance and impaired glucose tolerance in rats and that this association is not limited to high-fat diet rats (9). Epidemiological evidence is sparse. Short-term exposure to air pollution was linked to exacerbations of diabetes leading to death (10-14) and hospitalizations (13). Prevalence of diabetes was linked to air pollution $(14,15)$. Two prospective diabetes studies investigated the link with air pollution, with one reporting significant associations among a small number $(n=87)$ of women (16) and another failing to detect association in two large cohorts, except with a single traffic proximity proxy in women (17). Limited and mixed evidence precludes conclusions about causality between air pollution and diabetes and merits more study.

We studied the association between traffic-related air pollution levels at the residence and the risk for diabetes in an elderly Danish cohort and tested for an effect modification by lifestyle, education, and comorbid conditions. 


\section{RESEARCH DESICN AND METHODS}

\section{The Danish Diet, Cancer, and Health Cohort}

The Danish Diet, Cancer, and Health (DCH) cohort (18) consists of 57,053 people from Copenhagen or Aarhus aged 5065 years, recruited from 1993 to 1997. We linked the cohort members to the 1) Danish National Diabetes Register (NDR) for diabetes diagnoses; 2) the Central Population Registry to obtain date of death or emigration and detailed residential address history (1971-2006); 3) the Danish Address Database to obtain geographical coordinates of the addresses; and 4) the National Patient Register (dating back to 1976) to obtain data on hospital admissions for chronic obstructive pulmonary disease (COPD) (ICD-10, J40-44, and ICD-8, 490-92) and asthma (ICD-10, J45-46, and ICD-8, 493) before diabetes diagnoses.

\section{The Danish NDR}

The NDR was established in 2006 by the National Board of Health to describe and monitor the occurrence of diabetes in Denmark and provide data for epidemiological research (19). The NDR was established by linking existing nationwide administrative records in the Danish health care system: the National Patient Register, containing hospitals discharge diagnoses since 1994; the National Health Insurance Registry, with information of all services provided by general and specialist practitioners since 1973; and the Register of Medicinal Product Statistics, containing all prescriptions dispensed at Danish pharmacies since 1993. Inclusion criteria for the NDR were as follows: diabetes hospital discharge diagnoses in the National Patient Register defined as ICD-10: DE10-14, DH36.0, DO24, or ICD-8: 249, 250; chiropody for diabetic patients, five blood glucose measurements within 1 year, or two blood glucose measurements per year for 5 consecutive years, registered in the $\mathrm{Na}$ tional Health Insurance Registry; or second purchase of insulin or oral glucose-lowering drugs within 6 months, registered in the Register of Medicinal Product Statistics. The results of blood glucose measurements are not included in the NDR. Between 50 and $60 \%$ of the patients in NDR met more than one inclusion criterion. It was not possible to distinguish between type 1 and type 2 diabetes from NDR. Because of different dates of initiation of the underlying registers and accumulation of prevalent cases, only incidence values after 1 January 1995 were found to be reliable (19). Thus, the incidence of diabetes was defined as the earliest record in the diabetes register occurring after 1 January 1995, between baseline (19931997) and 27 June 2006. In addition to the original NDR definition including all cases, we defined a more strict incidence definition, including only confirmed diabetes, by excluding individuals who were included in the NDR solely because of a blood glucose test, since a number of these people probably did not have diabetes (19).

\section{Exposure assessment}

The Danish AirGIS human exposure modeling system (20) (described in the Supplementary Data) is a validated model (20) used to estimate outdoor levels of nitrogen dioxide $\left(\mathrm{NO}_{2}\right)$ and nitrogen oxide $\left(\mathrm{NO}_{\mathrm{x}}\right)$ at the residential addresses of the cohort members, who had residential history information available for more than $80 \%$ of the time between 1971 and 27 June 2006 (97.4\%). Missing values because of missing address or missing geographical coordinates were substituted by the levels calculated for the preceding address or, when the first address was missing, for the subsequent address, giving a complete series of annual mean $\mathrm{NO}_{2}$ and $\mathrm{NO}_{\mathrm{x}}$ concentrations since 1971. Mean $\mathrm{NO}_{2}$ levels since 1971 (calculated for each year of follow-up) was the main air pollution exposure proxy, but we also included the same measures for $\mathrm{NO}_{\mathrm{x}}$ (presented in the Supplementary Data), mean $\mathrm{NO}_{2}$ levels since 1991, 1-year mean $\mathrm{NO}_{2}$ at baseline, and 1-year mean $\mathrm{NO}_{2}$ at follow-up, traffic proximity (presence of a major road (density $\geq 10,000$ vehicles/day) within a 50-m radius of the baseline residence, and traffic load (total kilometers driven by vehicles) within a $100-\mathrm{m}$ radius of a baseline residence.

\section{Follow-up}

Cohort members were followed from date of recruitment or 1 January 1995 (start of the NDR) (whichever was last) until date of diabetes, as recorded in NDR, death, emigration, or 27 June 2006 (whichever came first). This was done separately for the two definitions of diabetes.

\section{Statistical analysis}

We used a Cox proportional hazards model to model the diabetes incidence, with age as the underlying timescale. The effects of exposure to $\mathrm{NO}_{2}$ on diabetes incidence was modeled as time-dependent variables and evaluated in several steps, with adjustment for a priori defined confounders: 1) adjusted only for age; 2) additionally adjusted for year (restricted cubic spline), to model the increase in diabetes incidence over follow-up time, and full adjustment for recognized diabetes risk factors: sex, BMI, waist-to-hip ratio, smoking status, smoking duration, smoking intensity, environmental tobacco smoke, educational level, leisure-time physical activity, alcohol intake, fruit consumption, and fat consumption; and 3) additionally adjusted for self-reported hypertension, hypercholesterolemia, and previous myocardial infarction (MI), which are risk factors for diabetes but are also associated with air pollution (6) and thus suspected to be on the biological pathway from air pollution to diabetes. Confounder definition is provided in the Supplementary Data. The potential modifiers of an effect between $\mathrm{NO}_{2}$ and diabetes by selected baseline confounders and comorbid conditions were evaluated by introducing interaction terms into the model and tested by the Wald test. Comorbidity with cardiovascular disease (hypertension, hypercholesterolemia, and MI) was self-reported at baseline, whereas comorbidity with COPD and asthma were defined by hospitalization for COPD or asthma, respectively, before baseline. The results are presented as hazard ratios (HRs) with 95\% CIs, per interquartile range (IQR) increase $\left(4.9 \mu \mathrm{g} / \mathrm{m}^{3}\right)$, estimated with survival library (R 2.9.0 statistical software). The graphical presentation of a functional form of an association between $\mathrm{NO}_{2}$ and confirmed diabetes was produced using restricted cubic spline in the design library ( $\mathrm{R} 2.9 .0$ statistical software).

RESULTS-Among the 57,053 cohort members, 571 were excluded because of a cancer diagnosis before enrollment, 2 with an uncertain date of cancer diagnosis, 960 with missing baseline address or missing geocode for baseline address, 1,341 with $\mathrm{NO}_{2}$ annual levels available for $<80 \%$ of the follow-up, 1,147 with self-reported diabetes at baseline, 173 with a diabetes record in the NDR before baseline, 6 with diabetes diagnoses from NDR between baseline and 1 January 1995, and 1,035 with missing information on covariates. Of the 51,818 eligible subjects followed over a mean of 9.7 years, $4,040(7.8 \%)$ developed diabetes in total (incidence rate 8.0 per 1,000 person-years), among which 1,163 included solely because of blood glucose measurements were excluded for a more strict definition of diabetes, resulting 
in 2,877 (5.5\%) diabetes cases (incidence rate 5.7 per 1,000 person-years).

Diabetic people were older; had higher BMI, waist-to-hip ratio, and alcohol and fat intake; had lower education; consumed less fruit; and were more likely to be men, current or previous smokers, exposed to environmental tobacco smoke, physically inactive, and have hypertension, hypercholesterolemia, or MI, but only marginally more likely to have COPD and asthma than nondiabetic people (Table 1). The estimated levels of $\mathrm{NO}_{2}$ varied greatly (Supplementary Fig. 1). Air pollution levels were higher for diabetic people than for the whole cohort (Table 1, Supplementary Table 1).

A statistically significant positive association was detected for both definitions of diabetes with all air pollution exposure proxies (except with an indicator of a major road within a 50-m radius of baseline residence for all diabetes) in models adjusted only for age, with a twice-as-strong effect for the confirmed compared with all diabetes cases (Table 2). There was no association between diabetes incidence and air pollution after adjustment for confounders when including all diabetes cases. For confirmed diabetes cases, full adjustment for lifestyle resulted in attenuated but borderline statistically significant association with mean levels of $\mathrm{NO}_{2}$ since 1971 (HR 1.04 [95\% CI 1.00-1.108] per IQR of $4.9 \mu \mathrm{g} / \mathrm{m}^{3}$ ), which was insensitive to further adjustment for hypertension, hypercholesterolemia, and MI. The

Table 1-Characteristics of the DCH cohort $(\mathrm{n}=51,818)$ by incident diabetes status at follow-up, for two definitions of diabetes incidence based on the NDR

Total cohort All diabetes* Confirmed diabetes*

\begin{tabular}{|c|c|c|c|}
\hline$n$ & 51,818 & 4,040 & 2,877 \\
\hline \multicolumn{4}{|l|}{ Baseline cohort covariates } \\
\hline Older than 56 years at baseline $[n(\%)]$ & $26,414(51.0)$ & $2,362(58.5)$ & $1,655(57.5)$ \\
\hline BMI [median (IQR)] & $25.5(4.9)$ & $28.6(5.9)$ & $29.1(5.9)$ \\
\hline Underweight $\left(\mathrm{BMI}<18.5 \mathrm{~kg} / \mathrm{m}^{2}\right)[n(\%)]$ & $406(0.8)$ & $9(0.2)$ & $6(0.2)$ \\
\hline Normal $\left(18.5 \leq \mathrm{BMI}<25 \mathrm{~kg} / \mathrm{m}^{2}\right)[n(\%)]$ & $22,475(43.4)$ & $751(18.6)$ & $401(13.9)$ \\
\hline Obese $\left(\mathrm{BMI} \geq 30 \mathrm{~kg} / \mathrm{m}^{2}\right)[n(\%)]$ & $7,299(14.1)$ & $1,504(37.2)$ & $1,208(42.0)$ \\
\hline Waist-to-hip ratio [median (IQR)] & $0.88(0.16)$ & $0.95(0.14)$ & $0.96(0.13)$ \\
\hline Waist-to-hip ratio $>0.90$ male $/>0.85$ female $[n(\%)]$ & $25,635(49.5)$ & $3,007(74.4)$ & $2,283(79.3)$ \\
\hline Never smoked $[n(\%)]$ & $18,438(35.6)$ & $1,202(29.7)$ & $823(28.6)$ \\
\hline Previously smoked $[n(\%)]$ & $14,822(28.6)$ & $1,273(31.5)$ & $891(31.0)$ \\
\hline Currently smoke $[n(\%)]$ & $18,558(35.8)$ & $1,565(38.7)$ & $1,163(40.4)$ \\
\hline Alcohol use (g/day) [median (IQR)] & $13.4(25.0)$ & $13.3(27.3)$ & $13.5(28.6)$ \\
\hline Physically active or play sports in leisure time $[n(\%)]$ & $28,282(54.6)$ & $1,784(44.2)$ & $1,185(41.2)$ \\
\hline Physical activity (h/week) [median (IQR)] & $2(2)$ & $2(2)$ & $2(2)$ \\
\hline$<8$ years of education $[n(\%)]$ & $16,961(32.7)$ & $1,618(40.0)$ & $1,209(42.0)$ \\
\hline $8-10$ years of education $[n(\%)]$ & $23,995(46.3)$ & $1,795(44.4)$ & $1,250(43.5)$ \\
\hline$>10$ years of education $[n(\%)]$ & $10,862(21.0)$ & $627(15.5)$ & $418(14.5)$ \\
\hline Fruit intake (g/day) [median (IQR)] & $145.2(165.3)$ & $142.5(164.4)$ & $136.8(162.1)$ \\
\hline Fat intake (g/day) [median (IQR)] & $81.2(37.7)$ & $81.9(38.0)$ & $82.2(38)$ \\
\hline Hypertension [n (\%)] & $8,200(15.8)$ & $1,226(30.3)$ & $915(31.8)$ \\
\hline Hypercholesterolemia [n (\%)] & $3,737(7.2)$ & $482(11.9)$ & $346(12.0)$ \\
\hline MI $[n(\%)]$ & $1,027(2.0)$ & $161(4.0)$ & $129(4.5)$ \\
\hline Traffic load $\ddagger$ within $100 \mathrm{~m}$ at baseline ( $10^{3}$ vehicle $\mathrm{km} /$ day) [median (IQR)] & $0.34(1.3)$ & $0.40(1.4)$ & $0.46(1.6)$ \\
\hline Major road $\S$ within $50 \mathrm{~m}$ at baseline $[n(\%)]$ & $4,184(8.1)$ & $373(9.2)$ & $287(10.0)$ \\
\hline
\end{tabular}

IQR, difference between 75th and 25th percentile. *All diabetes: based on original inclusion criteria in the NDR: hospital admission for diabetes, diabetes medication, reimbursement for chiropody due to diabetes, or glucose blood tests; confirmed diabetes: stricter definition of diabetes by exclusion of cases included solely because of glucose blood tests (not confirmed diabetes cases). † Defined as at least one hospital admission for COPD or asthma. $\ddagger$ Total number of kilometers traveled within $100 \mathrm{~m}$ (sum of product of street length and traffic density for each road). §Road with annual traffic density of $\geq 10,000$ vehicles. 
Table 2-Association between traffic-related air pollution per IQR increase in $\mathrm{NO}_{2}\left(4.9 \mu \mathrm{g} / \mathrm{m}^{3}\right)$ or traffic load within $100 \mathrm{~m}\left(1.2 \times 10^{3}\right.$ vehicles $\mathrm{km} /$ day) and diabetes among 51,818 DCH cohort participants, for two definitions of diabetes incidence based on the National Diabetes Register

Adjusted for age

Fully adjusted $\dagger$

Fully adjusted $\dagger+$ hypertension, hypercholesterolemia, and MI

All diabetes* $(n=4,040)$

$\mathrm{NO}_{2}\left(\mu \mathrm{g} / \mathrm{m}^{3}\right) 1971$ to follow-up

$\mathrm{NO}_{2}\left(\mu \mathrm{g} / \mathrm{m}^{3}\right) 1991$ to follow-up

$\mathrm{NO}_{2}\left(\mu \mathrm{g} / \mathrm{m}^{3}\right)$ at baseline (1 year)

Major road $\$$ within $50 \mathrm{~m}$

Traffic load $\S$ within $100 \mathrm{~m}\left(10^{3}\right.$ vehicle $\mathrm{km} /$ day $)$

$\mathrm{NO}_{2}\left(\mu \mathrm{g} / \mathrm{m}^{3}\right)$ at follow-up (1 year)

Confirmed diabetes* $(n=2,877)$

$\mathrm{NO}_{2}\left(\mu \mathrm{g} / \mathrm{m}^{3}\right) 1971$ to follow-up

$\mathrm{NO}_{2}\left(\mu \mathrm{g} / \mathrm{m}^{3}\right) 1991$ to follow-up

$\mathrm{NO}_{2}\left(\mu \mathrm{g} / \mathrm{m}^{3}\right)$ at baseline (1 year)

Major road $\ddagger$ within $50 \mathrm{~m}$

Traffic load $\S$ within $100 \mathrm{~m}\left(10^{3}\right.$ vehicle $\mathrm{km} /$ day $)$

$\mathrm{NO}_{2}\left(\mu \mathrm{g} / \mathrm{m}^{3}\right)$ at follow-up (1 year)
$1.06(1.03-1.09)$
$1.06(1.03-1.10)$
$1.03(1.00-1.06)$
$1.09(0.98-1.22)$
$1.03(1.00-1.05)$
$1.05(1.02-1.08)$

$1.11(1.07-1.15)$

$1.10(1.06-1.13)$

$1.08(1.05-1.11)$

$1.20(1.06-1.36)$

$1.05(1.03-1.08)$

$1.10(1.06-1.13)$
$1.00(0.97-1.03)$
$1.00(0.97-1.04)$
$0.98(0.95-1.01)$
$1.00(0.90-1.11)$
$1.00(0.98-1.02)$
$1.01(0.98-1.03)$

$1.04(1.00-1.08)$

$1.04(1.01-1.07)$

$1.02(0.98-1.05)$

$1.07(0.95-1.21)$

$1.02(1.00-1.04)$

$1.04(1.01-1.07)$
$1.00(0.97-1.04)$

$1.01(0.98-1.04)$

$0.98(0.95-1.01)$

$0.99(0.89-1.10)$

$1.00(0.98-1.02)$

$1.01(0.98-1.04)$

$1.04(1.00-1.08)$

$1.04(1.01-1.07)$

$1.02(0.98-1.05)$

$1.06(0.94-1.20)$

$1.02(1.00-1.04)$

$1.04(1.01-1.08)$

Data are HR (95\% CI). *All diabetes: based on original inclusion criteria in NDR: hospital admission for diabetes, diabetes medication, reimbursement for chiropody due to diabetes, or glucose blood tests; confirmed diabetes: stricter definition of diabetes by exclusion of cases included solely because of glucose blood tests (not confirmed diabetes cases). †Adjusted for sex, BMI, waist-to-hip ratio, smoking status, smoking duration, smoking intensity, environmental tobacco smoke, educational level, physical/sports activity in leisure time (indicated yes/no and intensity), alcohol consumption (indicated yes/no and intensity), fruit consumption, fat consumption, and calendar year. $\$$ Road with annual traffic density of $\geq 10,000$. $\S$ Total number of kilometers traveled within $200 \mathrm{~m}$ (sum of product of street length and traffic density for each road).

associations were identical with mean $\mathrm{NO}_{2}$ levels since 1991 (1.04 [1.01$1.07]$ ) and 1-year mean at end of followup (1.04 [1.01-1.08]), and weaker but borderline significant with traffic load within a 100-m radius of baseline residence (1.02 [1.00-1.04] per IQR of $1.2 \times 10^{3}$ vehicles $\mathrm{km} /$ day). Associations were weakly positive and statistically nonsignificant with 1 -year mean $\mathrm{NO}_{2}(1.02$ [0.98-1.05]) and indicator of a major road within a 50-m radius (1.06 [0.941.20]) of baseline residence. The findings for $\mathrm{NO}_{\mathrm{x}}$ were similar to those for $\mathrm{NO}_{2}$, but the associations were weaker (Supplementary Table 2). We found indication of linear dose response between mean $\mathrm{NO}_{2}$ levels since 1971 and diabetes incidence (Fig. 1).

We found enhanced associations between mean $\mathrm{NO}_{2}$ levels since 1971 and confirmed diabetes cases in women; people with a high waist-to-hip ratio $(>0.90$ for men and 0.85 for women); people with $<8$ years of education and without MI, hypertension, or hypercholesterolemia; and people with asthma or COPD, but no significant effect modifications were detected (Table 3). The association with $\mathrm{NO}_{2}$ was not modified by BMI. A significant effect modification was detected by physical activity (Wald test for interaction, $P=0.02$ ), with effects of $\mathrm{NO}_{2}$ only in physically active people
(HR 1.10 [95\% CI 1.03-1.16]), and by smoking status $(P=0.02)$, with enhanced effect of $\mathrm{NO}_{2}$ in nonsmokers (1.12[1.05-1.20]) and none in previous (1.03 [0.98-1.09]) or current smokers (1.00 [0.88-1.09]).

CONCLUSIONS - The risk for diabetes was weakly positively associated with increasing mean levels of traffic-related air pollution at the residence. The risk was highest in nonsmokers and physically active people.

This is the first study to relate air pollution to incidence of diabetes assessed objectively from a nationwide register. Previous studies used self-reports of diabetes incidence $(16,17)$, whereas diabetes prevalence was based on administrative databases of physician billing and hospital discharges (14) or on a national survey (15). The onset of diabetes based on self-reports is loosely defined and subject to recall bias $(16,17)$. Thus, the use of objective measures of diabetes onset based on the nationwide register, NDR, is appealing and convenient, since the entire population is covered by uniform inclusion criteria and the dropout rate is zero (19). The disadvantages of the NDR include inability to distinguish between diabetes types, although the majority of new diabetes cases in this age-group is most likely of type 2 diabetes (1). The date of inclusion in the NDR is only a proxy for the date of formal clinical diagnosis, which was likely made some time before inclusion in the register. No information on glucose or other clinical measurements used at diagnosis are available. Finally, the NDR likely underestimates the actual diabetes burden, since people without clinical diagnoses are not included.

"All diabetes" $(n=4,040)$ definition of incidence was previously validated (19) by a study comparing register-identified patients with their general practitioners, finding sensitivity of $86 \%$ and positive predictive value of $90 \%$. Of four inclusion criteria, hospital discharge diagnoses, diabetes medication records, and chiropody all reflect highly likely confirmed diabetes cases. In a "confirmed diabetes" definition $(n=$ 2,877 ), we excluded the 1,163 diabetes cases included solely because of blood glucose tests, because without available results of these tests, or other records in the NDR, it is likely that many of these people did not have diabetes. Because of increasing awareness among physicians on detecting undiagnosed diabetes, it is not uncommon for elderly healthy people to have five blood glucose tests per year.

Incidence rates in this cohort, as observed in all of Denmark, increased over the period 1995-2004 and showed a tendency to decline after 2004 (19), which we adjusted for by modeling the calendar year using restricted cubic splines. 


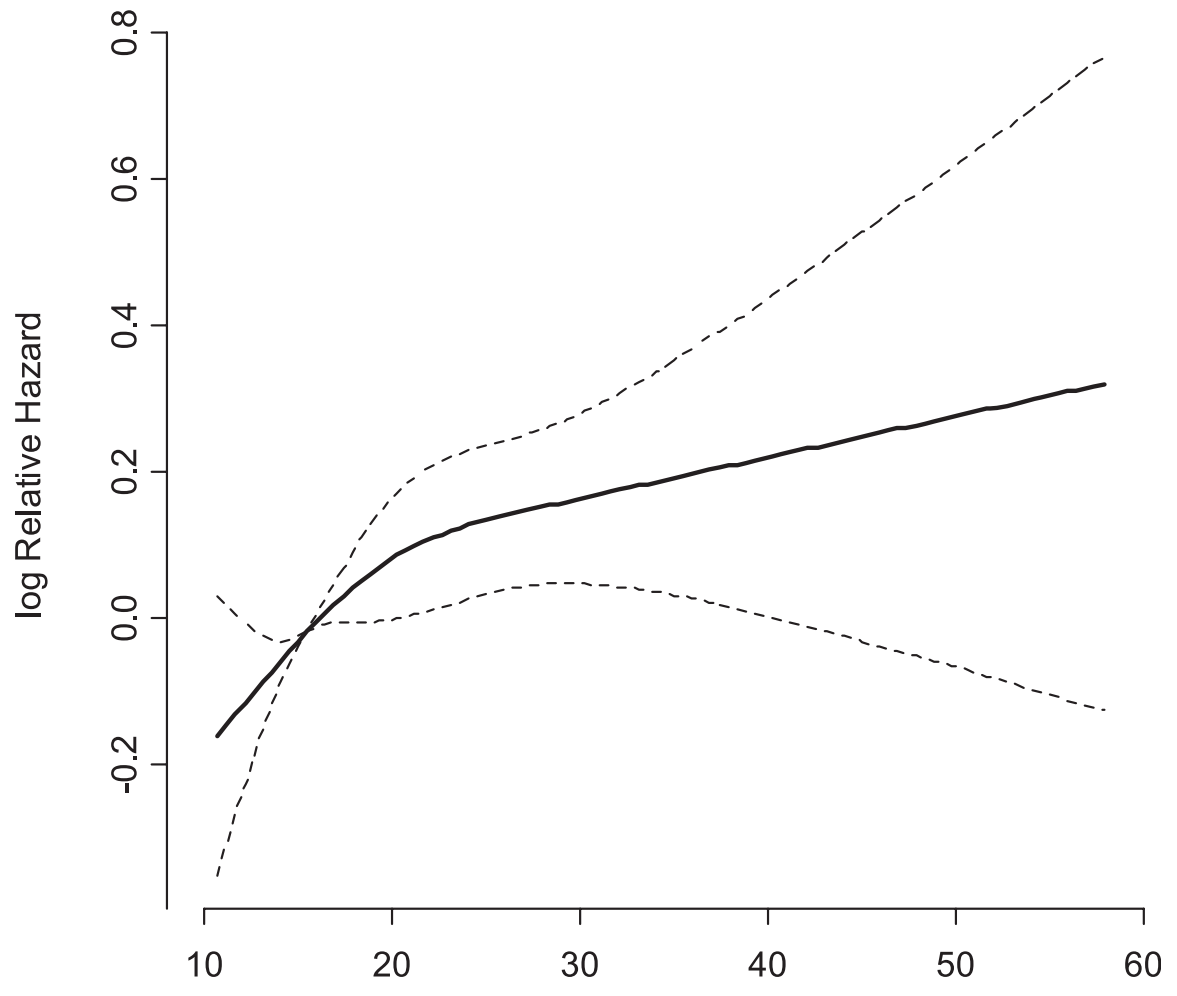

$\mathrm{NO} 2(\mu \mathrm{g} / \mathrm{m} 3)$

Figure 1-Association between exposure to $\mathrm{NO}_{2}$ levels at residence and incident diabetes defined by strict definition $(\mathrm{n}=2,877$ ) (log relative hazard with 95\% CI) for 51,818 Danish DCH cohort members, adjusted for sex, BMI, waist-to-hip ratio, smoking status (never, previous, current), smoking intensity, smoking duration, environmental tobacco smoke, physical activity, alcohol intake, fruit intake, fat intake, and educational level.

Our findings are generally consistent with the limited epidemiological evidence linking diabetes prevalence $(14,15)$ and the incidence $(16,17)$ to traffic-related air pollution. The magnitude of reported associations is smaller than that of other studies $(15,16)$, but these were based on women only. Moreover, proximity of the residence to a road was significantly associated with risk for diabetes only in a cohort of women (Nurses' Health Study), where no significant associations were detected with air pollution in a cohort of men (Health Professionals Follow-Up Study) (17). Our results also suggest that the risk for diabetes associated with air pollution may be limited to women (Table 3). Sex-related differences in susceptibility to air pollution could be associated with physiological differences in inflammatory responses or with lifestyle and activity patterns. Brook et al. (15) documented that women spent more time in and around the home and typically worked closer to home in Canada, contributing to smaller exposure misclassification and higher air pollution estimates, whereas no data in Denmark exist to support this.

Assigning individual exposure to air pollution with high spatial (addressspecific) and temporal (residential addresses) resolution, historically back to 1971, is novel and a major strength of this study. We detected identical associations with long (mean levels since 1971 and 1991) and short (1-year mean at follow-up) exposure windows to $\mathrm{NO}_{2}$. Puett et al. (17) on the other hand failed to detect associations with the 1-year mean particulate pollution levels before diabetes incidence. In our data, associations with baseline 1-year mean $\mathrm{NO}_{2}$ levels and more "naïve" proxies based on traffic density around the residence were weaker, likely because of exposure misclassification in individuals who moved since baseline.

The association between air pollution and diabetes incidence in his cohort was strongest in nonsmokers (Table 3). Smoking and environmental tobacco smoke are confirmed risk factors for type 2 diabetes (21), as corroborated by our (Table 1 ) and previous data $(16,17)$. Inhalation of tobacco smoke triggers similar responses in the lung as air pollution, and these two related exposures share a plausible biological mechanism leading to glucose intolerance via systemic inflammation and inflammation in adipose tissue (22). It has been hypothesized that preexisting low-level inflammation seen in smokers would further enhance the effects of air pollution (2). In contrast, our results suggest a marginal contribution of air pollution to the development of diabetes in smokers and ex-smokers.

Furthermore, in contrast to existing evidence suggesting that individuals with preexisting cardiovascular disease would be more susceptible to the adverse effects of air pollution $(2,7,8)$, we found no statistically significant effect modification of the effects of air pollution by MI, hypertension, or hypercholesterolemia (Table 3). This finding contradicts the study of air pollution-triggered diabetes deaths, which reported effects only in individuals with preexisting cardiovascular disease (11). Whereas diabetic patients have been found to be at higher risk of developing cardiovascular disease due to air pollution than nondiabetic people (7), our results do not support the idea that cardiovascular disease enhances the susceptibility to air pollution and the associated risk of diabetes. Similarly, physical inactivity was found to be the expected risk factor for diabetes, whereas we only found association with air pollution among individuals who were physically active (Table 3). This observation may reflect smaller air pollution exposure misclassification in physically active individuals, because of more time spent outdoors and higher relative exposures and exaggerated air exchange rates during physical activity. It is possible that the association between diabetes and air pollution is only detected among individuals with a low prior risk, i.e., people who are physically active, representing a limited absolute risk, whereas this cannot be detected among individuals with a priori increased risk, suggesting additive patterns (23). Among people with a low level of education, we found the expected increased risk for diabetes as well as enhanced effects of air pollution, although without significant interaction.

We have earlier reported significant positive association between hospital admissions for COPD and asthma and long-term exposure to $\mathrm{NO}_{2}$ in this cohort $(24,25)$. It can thus be argued that our results may be affected by diagnosis bias, due to more frequent screening for disease, including diabetes, among chronic respiratory disease patients, who are also more likely to 
Table 3-Modification of associations* between $\mathrm{NO}_{2}$ (1971 follow-up, per IQR of $\left.4.9 \mu \mathrm{g} / \mathrm{m}^{3}\right)$ and confirmed diabetes cases $(\mathrm{n}=2,877)$ by baseline characteristics and comorbid conditions among $51,818 \mathrm{DCH}$ cohort participants

Incidence rate $\dagger$

$\operatorname{HR}(95 \% \mathrm{CI})$

$P \ddagger$

BMI

$\begin{array}{lrrlll}\text { Normal/underweight }\left(\mathrm{BMI}<25 \mathrm{~kg} / \mathrm{m}^{2}\right) & 407 & 1.8 & 1.10(1.00-1.21) & \\ \text { Overweight }\left(25 \leq \mathrm{BMI}<30 \mathrm{~kg} / \mathrm{m}^{2}\right) & 1,262 & 6.0 & 1.01(0.96-1.07) & \\ \text { Obese }\left(\mathrm{BMI} \geq 30 \mathrm{~kg} / \mathrm{m}^{2}\right) & 1,208 & 18.3 & 1.05(1.00-1.12) & 0.30\end{array}$

Waist-to-hip ratio

$\begin{array}{lrrrr}>0.90 / 0.85 \text { (male/female) } & 2,283 & 9.5 & 1.09(1.01-1.18) & \\ \leq 0.90 / 0.85 \text { (male/female) } & 594 & 2.3 & 1.04(1.00-1.08) & 0.24\end{array}$

Sex

$\begin{array}{llll}\text { Males } & 1,702 & 7.3 & 1.01(0.97-1.07)\end{array}$

Females

1,175

4.4

$1.07(1.01-1.13)$

0.15

Physical activity

$\begin{array}{lllll}\text { Yes } & 1,185 & 4.3 & 1.10(1.03-1.16) & \\ \text { No } & 1,692 & 7.5 & 1.00(0.95-1.05) & 0.02\end{array}$

Smoking status

$\begin{array}{lrrrr}\text { Never } & 823 & 4.5 & 1.12(1.05-1.20) & \\ \text { Previous } & 891 & 6.2 & 1.02(0.95-1.10) & \\ \text { Current } & 1,163 & 6.5 & 1.00(0.95-1.06) & 0.05\end{array}$

Education

$<8$ years of education $\quad 1,209$

$8-10$ years of education

1,250

$\geq 10$ years of education

418

MI

Yes

129

2,748

14.8

7.4

$1.06(1.01-1.12)$

5.4

$1.03(0.98-1.09)$

$0.98(0.88-1.09) \quad 0.44$

No

915

Yes

7,285

12.1

4.6

$1.03(0.96-1.10)$

No

7.285

Hypercholesterolemia

Yes

No

COPD

$\begin{array}{lr}\text { Yes } & 140 \\ \text { No } & 2,737\end{array}$

Asthma

Yes

78

No

2,799

346
2,531

10.0

5.4

$0.96(0.85-1.09)$

1.05 (1.01-1.09)

0.20

*Adjusted for sex, BMI, waist-to-hip ratio, smoking status, smoking duration, smoking intensity, environmental tobacco smoke, educational level, physical/sports activity in leisure time (indicated yes/no and intensity), alcohol consumption (indicated yes/no and intensity), fruit consumption, fat consumption, and calendar year. $†$ Crude rate per 1,000 person-years. $\$$ For Wald or likelihood ratio test for interaction.

live in areas with higher air pollution levels than the rest of the cohort $(24,25)$. However, this is unlikely, since we found only a marginally higher prevalence of asthma and COPD in diabetic people than in nondiabetic people (Table 1), and history of asthma or COPD hospitalizations was not significantly associated with increased risk of diabetes (results not shown). Furthermore, comorbidity with asthma or COPD did not statistically significantly modify the effect of air pollution on diabetes (Table 3).

Effects of air pollution were slightly enhanced in people with a high waist-tohip (Table 3), corroborating the evidence from animal models, where exposure to air pollution led to glucose intolerance only in obese rats (7). However, enhanced air pollution effects were not seen with increasing BMI. This is the first study of air pollution and diabetes to adjust for waist-to-hip ratio, which is an independent predictor of diabetes next to BMI. Excluding waist-to-hip ratio from the full model as others did (14-17) resulted in even stronger associations with 1.10]) as did exclusion of BMI (1.08 [1.041.12]). Mean levels of $\mathrm{NO}_{2}$ were weakly correlated with BMI (correlation coefficient $r=0.03)$ and waist-to-hip ratio $(r=0.02)$. air pollution (HR 1.05 [95\% CI 1.02-
Obesity, the most important risk factor for diabetes (1), has been linked to air pollution (22) and may be on the biological pathway between air pollution and diabetes. Our results remained unchanged when adding total energy intake, including energy due to alcohol intake, into the model.

The strengths of this study include the large prospective cohort with an objective assessment of diabetes incidence and welldefined confounders, with minimal possibility of recall and information bias. The main limitation is the exposure misclassification in modeled air pollution concentrations, since these are only proxies of personal exposure. This cohort lacked information on indoor exposures, use of air purifier and air conditioning, work address, working time, as well as commuting habits and personal outdoor activity patterns. However, lack of these parameters and resulting exposure misclassification is likely to be nondifferential with respect to diabetes diagnoses. The air pollution models used to assess $\mathrm{NO}_{2}$ levels were successfully validated $(26,27)$ and applied in epidemiological studies $(24,25)$. Another limitation is the lack of results from blood glucose tests from the National Health Insurance Registry, which precludes better ascertainment of diabetes among 1,163 people included in NDR solely due to blood glucose tests and more specific overall diabetes definition. Finally, there is little evidence coming from original research that supports biological plausibility of our findings $(8,9,22)$, and more studies will be needed to confirm or reject the link between air pollution and diabetes.

We detect weak positive associations between diabetes incidence and trafficrelated air pollution at residence, and we add to the body of evidence that air pollution may be a risk factor for diabetes. We offer several novel findings that need to be reproduced. The effects of air pollution were strongest in nonsmokers and physically active people. Facing the emerging challenges in controlling the diabetes epidemic, a possibility that air pollution contributes to the diabetes burden may have a huge public health impact.

Acknowledgments - No potential conflicts of interest relevant to this article were reported.

Z.J.A. takes full responsibility for the work as a whole, including the study design, access to data, and the decision to submit and publish the article. Z.J.A. analyzed data, designed the study, and wrote the manuscript. O.R.-N. contributed to the design of the study and preparation of the manuscript. M.K., S.S.J., 
and M.H. developed the Danish dispersions air pollution model and calculated air pollution data. S.L. secured the funding for the study and contributed to the design and interpretation of findings. A.T. and K.O. are the main investigators of the DCH cohort study. M.S. designed the study, contributed to the analysis strategy, and helped in preparing the manuscript.

The authors thank Lissa Churchward, Institute of Cancer Epidemiology, Danish Cancer Society, for editing the manuscript.

\section{References}

1. van Dieren S, Beulens JW, van der Schouw YT, Grobbee DE, Neal B. The global burden of diabetes and its complications: an emerging pandemic. Eur J Cardiovasc Prev Rehabil 2010;17(Suppl. 1):S3-S8

2. Bhatnagar A. Could dirty air cause diabetes? Circulation 2009;119:492-494

3. Lockwood AH. Diabetes and air pollution Diabetes Care 2002;25:1487-1488

4. Zanobetti A, Schwartz J. Are diabetics more susceptible to the health effects of airborne particles? Am J Respir Crit Care Med 2001;164:831-833

5. Sarwar N, Gao P, Seshasai SR, et al. Diabetes mellitus, fasting blood glucose concentration, and risk of vascular disease: a collaborative meta-analysis of 102 prospective studies. Lancet 2010;375: 2215-2222

6. Brook RD, Rajagopalan S, Pope CA 3rd, et al. Particulate matter air pollution and cardiovascular disease: an update to the scientific statement from the American Heart Association. Circulation 2010;121: 2331-2378

7. Wellen KE, Hotamisligil GS. Inflammation, stress, and diabetes. J Clin Invest 2005; 115: 1111-1119

8. Sun Q, Yue P, Deiuliis JA, et al. Ambient air pollution exaggerates adipose inflammation and insulin resistance in a mouse model of diet-induced obesity. Circulation 2009;119:538-546
9. Xu X, Liu C, Xu Z, et al. Long-term exposure to ambient fine particulate pollution induces insulin resistance and mitochondrial alteration in adipose tissue. Toxicol Sci 2011 Aug 27. [Epub ahead of print]

10. Kan H, Jia J, Chen B. The association of daily diabetes mortality and outdoor air pollution in Shanghai, China. J Environ Health 2004;67:21-26

11. Goldberg MS, Burnett RT, Yale JF, Valois MF, Brook JR. Associations between ambient air pollution and daily mortality among persons with diabetes and cardiovascular disease. Environ Res 2006;100:255267

12. Maynard D, Coull BA, Gryparis A, Schwartz J. Mortality risk associated with short-term exposure to traffic particles and sulfates. Environ Health Perspect 2007;115:751755

13. Zanobetti A, Franklin M, Koutrakis P, Schwartz J. Fine particulate air pollution and its components in association with cause-specific emergency admissions. Environ Health 2009;8:58

14. Pearson JF, Bachireddy C, Shyamprasad S, Goldfine AB, Brownstein JS. Association between fine particulate matter and diabetes prevalence in the U.S. Diabetes Care 2010;33:2196-2201

15. Brook RD, Jerrett M, Brook JR, Bard RL, Finkelstein MM. The relationship between diabetes mellitus and traffic-related air pollution. J Occup Environ Med 2008; 50:32-38

16. Krämer U, Herder C, Sugiri D, et al. Traffic-related air pollution and incident type 2 diabetes: results from the SALIA cohort study. Environ Health Perspect 2010;118:1273-1279

17. Puett RC, Hart JE, Schwartz J, Hu FB, Liese AD, Laden F. Are particulate matter exposures associated with risk of type 2 diabetes? Environ Health Perspect 2010 Nov 30. [Epub ahead of print]

18. Tjønneland A, Olsen A, Boll K, et al. Study design, exposure variables, and socioeconomic determinants of participation in Diet,
Cancer and Health: a population-based prospective cohort study of 57,053 men and women in Denmark. Scand J Public Health 2007;35:432-441

19. Carstensen B, Kristensen JK, Ottosen P, Borch-Johnsen K; Steering Group of the National Diabetes Register. The Danish National Diabetes Register: trends in incidence, prevalence and mortality. Diabetologia 2008;51:2187-2196

20. Jensen SS, Berkowicz R, Hansen SH, Hertel O. A Danish decision-support GIS tool for management of urban air quality and human exposures. Transp Res Part D Transp Environ 2001;6:229-241

21. Kowall B, Rathmann W, Strassburger K, et al. Association of passive and active smoking with incident type 2 diabetes mellitus in the elderly population: the KORA S4/F4 cohort study. Eur J Epidemiol 2010;25:393-402

22. Harrington M. Air pollution linked to obesity, inflammation. Lab Anim (NY) 2011; $40: 3$

23. Rothman KJ, Poole C. A strengthening programme for weak associations. Int J Epidemiol 1988;17:955-959

24. Andersen ZJ, Hvidberg M, Jensen SS, et al. Chronic obstructive pulmonary disease and long-term exposure to traffic-related air pollution: a cohort study. Am J Respir Crit Care Med 2011;183:455-461

25. Andersen ZJ, Bønnelykke K, Hvidberg M, et al. Long-term exposure to air pollution and asthma hospitalizations in elderly adults: a cohort study. Thorax 2 September 2011 [Epub ahead of print]

26. Ketzel M, Berkowitz R, Hvidberg M, Jensen SS, Raaschou-Nielsen O. Evaluation of AirGIS: a GIS-based air pollution and human exposure modelling system. IJEP. In press

27. Kakosimos KE, Hertel O, Ketzel M, Berkowicz R. Operational Street Pollution Model (OSPM): a review of performed application and validation studies, and future prospects. Environ Chem 2010;7: 485-503 